\title{
Educación Científica con enfoque Ciencia-Tecnología- Sociedad-Ambiente. Construcción de un Instrumento de Análisis de las Directrices Curriculares
}

\author{
Isabel M. Fernandes ${ }^{(1,2)}$, Delmina M. Pires ${ }^{(1)}$ y Rosa M. Villamañán ${ }^{(2)}$ \\ (1) Departamento de Ciências da Natureza, Instituto Politécnico de Bragança, Escola Superior de \\ Educação Campus de Santa Apolónia, Apartado 1101, 5301-856 Bragança, Portugal. \\ (e-mail: isabel.fernandes@ipb.pt; piresd@ipb.pt) \\ (2) Departamento de Didáctica de las Ciencias Experimentales, Sociales y de la Matemática, Universidad \\ de Valladolid, Facultad de Educación y Trabajo Social. Paseo Belén, 1, 47011 Valladolid, España. \\ (e-mail: rvillama@dce.uva.es)
}

Recibido Jun. 17, 2014; Aceptado Jul. 10, 2014; Versión final recibida Sep. 4, 2014

\begin{abstract}
Resumen
El objetivo principal del enfoque Ciencia-Tecnología-Sociedad-Ambiente (CTSA) es el desarrollo de la cultura científica en los estudiantes, preparándolos para el ejercicio de una ciudadanía activa y consciente. Esta perspectiva de enseñanza, debe ser considerada en las directrices curriculares, así como en otros documentos que regulan la acción educativa. Se ha construido un instrumento de análisis con objeto de determinar si la perspectiva CTSA está integrada en los documentos curriculares. Su construcción se ha efectuado en cinco etapas fundamentándose en datos y consideraciones de investigaciones recientes en didáctica de las ciencias, así como en diversos instrumentos ya utilizados en el ámbito CTSA. Este instrumento se ha presentado, debatido públicamente y reformulado según las sugerencias recibidas, y ha sido validado por expertos. Se concluye que la propuesta es una herramienta eficaz para alcanzar los objetivos propuestos.
\end{abstract}

Palabras clave: directrices curriculares, perspectiva CTSA, cultura científica, didáctica de las ciencias.

\section{Scientific Education with the Approach Environment, Science, Technology and Society. Development of an Instrument for the Analysis of Curriculum Guidelines}

\begin{abstract}
The main objective of the Environment, Science, Technology and Society approach (ESTS) in science education is to develop scientific literacy in students, preparing them for the exercise of active and conscious citizenship. This view of education must be considered in curriculum guidelines and other documents governing the educational activity. An analytical tool to determine if the ESTS perspective is integrated into the curriculum documents has been developed. Its construction has been done in five stages on the basis of data and considerations of recent research in science education, as well as various tools already used in the ESTS perspective. This instrument has been publicly presented, debated and reformulated according to the suggestions received, and has been validated by experts. It is concluded that the proposal represents an effective tool to achieve the established objectives.
\end{abstract}

Keywords: curriculum guidelines, ESTS perspective, scientific literacy, science didactics. 


\section{INTRODUCIÓN}

En las últimas décadas el enfoque CTS se ha consolidado como una propuesta para el desarrollo de los currículos de ciencias de muchos países. El interés por este enfoque curricular se remonta a las décadas de los ochenta y noventa cuando se llevaron a cabo varios proyectos con recomendaciones y propuestas curriculares para la enseñanza de las ciencias con objeto de fomentar la cultura científica de los estudiantes. Entre ellos, podemos citar: Project 2061 - Science for all americans (AAAS, 1990); Benchmarks for Science Literacy (AAAS, 1993); National Science Education Standards (NRC, 1996); y Beyond 2000 Science Education for the future (Millar y Osborne, 1998). Por otra parte, varias y prestigiosas organizaciones internacionales como, por ejemplo, la Organización de los Estados Iberoamericanos (OEI, 2001), la National Science Education Standards (NRC, 1996) y la American Association for the Advancement of Science (AAAS, 1993), han propuesto recomendaciones para adaptar los currículos de ciencias a la sociedad actual, reforzando la necesidad de proveer a los profesores de orientaciones que les permitan implementar prácticas pedagógicas promotoras del desarrollo de la cultura científica en los estudiantes.

La inclusión de la letra A de Ambiente a las siglas CTS contribuye a dar una imagen más completa y contextualizada de la ciencia y supone considerar la comprensión de cuestiones ambientales y de calidad de vida. De este modo, este enfoque CTSA (Ciencia-Tecnología-Sociedad-Ambiente) pretende, sentar las bases para un futuro sostenible (Gil y Vilches, 2004; Vilches y Gil-Pérez, 2010). Esta forma de enfocar la ciencia puede constituir una vía eficaz para promover la cultura científica, entendida como la capacidad para comprender los avances científico-tecnológicos de la sociedad actual. Esto contribuiría al ejercicio de una ciudadanía activa y consciente, así como el desarrollo de competencias que posibiliten utilizar los conocimientos adquiridos en la escuela al contexto cotidiano. La cultura científica requiere el aprendizaje tanto de contenidos de ciencias como sobre su naturaleza, o sea, además de los conocimientos y procedimientos básicos de la ciencia, los estudiantes deberán también adquirir conocimientos y actitudes sobre lo que es la ciencia, como se construye y funciona, cuáles son sus relaciones con la tecnología, con la sociedad y con el ambiente (Bennássar et al., 2010; Hodson 2008; Lederman, 2007 citados por GarcíaCarmona y Criado, 2012).

Debido a ello, consideramos que en una sociedad altamente tecnológica como la actual, donde los avances científicos son casi diarios, la educación en ciencias con orientación CTSA además de una exigencia, es una necesidad (Fernandes y Pires, 2013). Las potencialidades de esta perspectiva de enseñanza de la ciencia están reconocidas para el desarrollo de ciudadanos informados y críticos en temas de ciencia y tecnología (Alves, 2011; Vieira, Tenreiro-Vieira y Martins, 2011). Por ello, debería ser tenida en cuenta de forma clara y explícita en los currículos de ciencias y en los documentos que regulan la actuación de los profesores a nivel de aula. El desinterés que los estudiantes manifiestan con relación a la ciencia y a los temas científicos ha sido motivo de debate en la comunidad científica y objeto de diversos estudios (Eurydice, 2011, 2006; Martins et al., 2011; Osborne y Dillon, 2008; Rocard et al., 2007), que han puesto de manifiesto la necesidad de revisar y rediseñar los currículos de ciencias para proporcionar una educación científica contextualizada y adecuada a los intereses y necesidades de la sociedad actual. Con objeto de aumentar la motivación e interés por la ciencia, Eurydice (2011), por ejemplo, considera esencial que el currículo enfatice las conexiones con las experiencias personales de los estudiantes y contemple las relaciones entre la ciencia y la tecnología, así como, cuestiones sobre la sociedad contemporánea, además de proporcionar la posibilidad de discutir los aspectos filosóficos de la ciencia y los relacionados con la sostenibilidad del planeta y la calidad de vida.

\section{CAMBIO DE LAS DIRECTRICES CURRICULARES}

Basado en los antecedentes presentados en la sección anterior, es necesario cambiar los currículos, para hacerlos más atractivos, así como para adaptarlos a las necesidades de los estudiantes, y a los avances científico-tecnológicos que caracterizan la sociedad actual, para la cual los ciudadanos deben estar preparados. Sólo los individuos críticos, autónomos y portadores de competencias, tales como la capacidad de resolver problemas en contextos reales, serán capaces de integrarse plenamente en la sociedad donde viven, es decir, podrán tomar decisiones responsables e informadas en un mundo cada vez más complejo, y comprender las consecuencias de sus actos, decisiones y opciones. En este contexto, el actual paradigma de la enseñanza de las ciencias se ha debatido con un problema adicional: enseñar sobre la naturaleza de la ciencia (historia, filosofía, sociología...) y las relaciones que se establecen con la tecnología, la sociedad y el ambiente. Sin embargo, estas cuestiones son complejas y no están claras en los currículos de ciencias (García-Carmona y Criado, 2012). Por ello, diversas investigaciones en el ámbito de la educación CTSA (Fernandes y Pires, 2013; Prieto et al., 2012) se han preocupado por las siguientes cuestiones: ¿Por qué enseñar ciencia? ¿Qué ciencia se debe enseñar? ¿Cómo se debe enseñar la ciencia?...que deben orientar la construcción de los currículos de ciencias. 


\section{¿Por qué enseñar ciencia?}

La gran meta educativa del enfoque CTSA, como hemos indicado, es el desarrollo de la cultura científica en los estudiantes, que les permita comprender los avances científico-tecnológicos de la sociedad actual, haciendo que sean capaces de utilizar en contextos reales los conocimientos y las competencias adquiridas en la escuela. A pesar de existir diversas definiciones e interpretaciones del concepto de cultura científica (Aikenhead, 2009; DeBoer, 2000 citado por Vieira, 2007; León et al., 2013), es posible encontrar aspectos comunes en su definición. La cultura científica es entendida esencialmente como el dominio del conocimiento científico y la capacidad del pensamiento crítico sobre una determinada situación, así como la capacidad de aplicar este conocimiento para resolver problemas. Incluye la capacidad de reflexión y saber hacer uso de la información científica para generar opiniones, actitudes críticas y hacer frente a la vida diaria (Díaz y García, 2011). Como señalan León et al., 2013 (apoyados en estudios de Martín, Vergara y Fuentes, 2011), en una sociedad altamente científica y tecnológica, el concepto de cultura científica se puede entender como el correspondiente a un conjunto de significados, expectativas y comportamientos sobre la ciencia y la tecnología evidenciados y compartidos, local o globalmente, por los individuos de un determinado grupo social.

Pero, para implementar una educación CTSA capaz de promover la cultura científica, es necesario que los currículos de ciencias propongan: (a) el desarrollo de procedimientos científicos (observar, inferir, clasificar, explicar, relacionar, argumentar...); (b) la resolución de problemas; (c) la mejora del pensamiento crítico; (d) el desarrollo de principios y normas de conducta responsables y conscientes, individuales y colectivas; (e) la toma de decisiones conscientes, informadas y argumentadas frente a las consecuencias de la acción humana en el ambiente; y (f) el poderse desenvolver en cuestiones problemáticas actuales relacionadas con la ciudadanía, la sostenibilidad y la protección del ambiente.

\section{¿Qué ciencia se debe enseñar?}

La enseñanza de las ciencias, requiere la necesidad de considerar la ciencia y la tecnología como dos sistemas que interactúan intelectual y socialmente, así como la necesidad de seleccionar problemas y ejemplos de la vida cotidiana y no una enseñanza que enfatice la ciencia "pura", básica y descontextualizada (Prieto et al., 2012). Este propósito exige la contextualización simultánea de los aspectos científicos, tecnológicos y sociales. Para mejorar la enseñanza de la ciencia según la perspectiva CTSA se necesita: (a) dar prioridad al aprendizaje de conceptos que sean importantes y relevantes para las necesidades de los estudiantes, para el progreso social y para el bien común, centrando la enseñanza en temas científicos socio-ambientales relevantes y polémicos; (b) promover el aprendizaje de los conceptos científicos a partir de ejemplos de su vida diaria, conectando el conocimiento científico con el conocimiento cotidiano, involucrando a los estudiantes en aprendizajes significativos y contextualizados, necesarios para comprender el mundo en su globalidad y complejidad; (c) comprender los aspectos epistemológicos y sociológicos de la construcción de la ciencia, llevando a los estudiantes a reconocer las ventajas y las limitaciones de la ciencia y de la tecnología; y (d) conocer, valorar y usar la tecnología en su vida personal, así como ser capaz de contrastar las explicaciones científicas con las ideas del sentido común (Fernandes y Pires, 2013).

Los currículos de ciencias deben permitir desarrollar en los estudiantes conocimientos científicos considerados esenciales para su formación personal y social, efectuando un enfoque contextualizado con temas actuales relacionados con sus conocimientos previos y con su vida diaria. Se precisa también que propongan: (a) discusiones sobre temas científicos en función de su utilidad social; (b) situaciones en las que diferentes realidades sociales sean el origen de nuevos descubrimientos científicos e innovaciones tecnológicas (relación sociedad-tecnología); (c) abordar las ventajas y las limitaciones del conocimiento científico-tecnológico, así como sus impactos en la sociedad y en el ambiente (relación ciencia-tecnologíasociedad-ambiente); (d) poner en evidencia las relaciones recíprocas entre la ciencia y la tecnología (relación ciencia-tecnología); (e) destacar los cambios en las condiciones de vida de las personas relacionadas con los avances tecnológicos a lo largo del tiempo (relación sociedad-tecnología); (f) enfatizar los impactos de la sociedad y del ambiente en los avances científico-tecnológicos (relación cienciatecnología-sociedad-ambiente); (g) dar prioridad a tratar la exploración de contenidos científico-tecnológicos relacionados con otros campos del saber donde se exige la comprensión de las interrelaciones CTSA; (h) debatir datos relacionados con la naturaleza y la historia de la ciencia y/o diferentes visiones del conocimiento científico a lo largo del tiempo; (i) presentar el conocimiento de una forma no dogmática; (j) informar sobre el trabajo y función del científico; y (k) discutir las posibles presiones sociales, políticas, religiosas o económicas que pueda sufrir la ciencia (dimensión filosófica, histórica, psicológica y sociológica de la ciencia). 


\section{¿Cómo se debe enseñar la ciencia?}

En los últimos años, los aspectos relacionados con la eficacia de las diferentes estrategias didácticas en la enseñanza de las ciencias, han sido la gran preocupación de muchas investigaciones en Didáctica de las Ciencias con enfoque CTSA (Alves, 2011; Fernandes y Pires, 2013; Gonzalez y Rasilla, 2011; Manassero, Roig, Bonnin y Moralejo, 2013). Sin embargo, consideramos que no existe un modelo único de enseñanza perfecto, capaz de solucionar todos los problemas educativos, es decir, no existe ninguna estrategia didáctica que funcione con todos los estudiantes, con todos los temas, en todos los contextos, y satisfaga todos los objetivos. Sea cual sea la práctica didáctica que cada profesor defienda, nos parece que, en cualquier circunstancia, deberá incluir un conjunto de estrategias diversificadas todas ellas fundamentadas en una perspectiva socio-constructivista, entre las cuales deberán estar la resolución de problemas, el debate, el dialogo y la argumentación en el aula. Por ello, los currículos de ciencias deberían sugerir a los profesores la utilización de procedimientos metodológicos para incentivar al estudiante a: (a) utilizar diferentes recursos dentro y fuera del aula; (b) proponer la realización de actividades prácticas, experimentales, de laboratorio y salidas de campo donde sea posible realzar y explorar las relaciones CTSA; y (c) involucrar activamente al estudiante en actividades de debates, resolución de problemas, discusiones, investigaciones sobre cuestiones donde esas relaciones e interacciones se manifiesten.

\section{METODOLOGIA}

Debido a la gran importancia del enfoque CTSA en la enseñanza de las ciencias, hemos construido un instrumento de análisis, aplicable a las directrices y documentos curriculares, para poner de manifiesto si contemplan las recomendaciones dadas por esta perspectiva de enseñanza de las ciencias y si fomentan su implementación en el contexto del aula.

\section{Construcción del instrumento de análisis: etapas}

La construcción del instrumento de análisis se ha efectuado en cinco etapas distintas: (a) revisión bibliográfica sobre el paradigma didáctico actual de la enseñanza de las ciencias con enfoque CTSA (Ciencia, Tecnología, Sociedad y Ambiente); (b) evaluación de los instrumentos de análisis de Silva (2007) y Pereira (2012) en el ámbito de la educación en las ciencias; (c) estudio del cuestionario Views on ScienceTechnology-Society - VOSTS - (Aikenhead et al., 1989); (d) análisis del Cuestionario de Opiniones sobre Ciencia, Tecnología y Sociedad - COCTS - (Manassero et al., 2001, 2003); y (e) validación por un grupo de jueces expertos en Didáctica de las Ciencias.

La revisión bibliográfica efectuada tuvo en cuenta las diversas recomendaciones nacionales e internacionales para la educación en ciencias con enfoque CTSA, y los cuestionarios VOSTS y COCTS que han constituido los principios orientadores. El cuestionario de respuesta múltiple VOSTS es un instrumento desarrollado empíricamente, a partir de entrevistas y cuestionarios realizados a estudiantes y profesores. Sus respuestas han sido sintetizadas en frases que constituyen las diferentes opciones y, por ello, posiblemente, este instrumento es el que mejor caracteriza las concepciones sobre ciencia, tecnología, sociedad y sus interrelaciones. Las cuestiones que integran este cuestionario incluyen diferentes dimensiones conceptuales, tales como, definiciones de ciencia y tecnología; interacciones entre ciencia, tecnología y sociedad; sociología externa de la ciencia (relaciones CTSA), sociología interna de la ciencia y naturaleza del conocimiento científico. Por todo ello, es considerado por Vázquez et al., 2010 (citado en Rodrigues y Vieira, 2012) muy útil en la identificación de las concepciones de los profesores y estudiantes sobre la ciencia, su naturaleza y sus relaciones con otras áreas del saber. El cuestionario COCTS es considerado por Acevedo Díaz et al. (2007) y Filho et al. (2013) como una adaptación al contexto español del VOSTS y del Teacher's Belief about Science-Technology-Society [TBA-STS] (Rubba y Harkness, 1993; Rubba, Schoneweg-Bradford y Harkness, 1996). Es un cuestionario de respuesta múltiple diseñado para investigar las ideas de los profesores sobre los temas CTS, incluyendo también dimensiones relativas a definiciones de ciencia, tecnología, sociología interna de la ciencia, sociología externa de la ciencia y epistemología de la ciencia. Dado el enorme potencial de los cuestionarios VOSTS y COCTS, ambos fueron consultados con detalle. Este examen minucioso ha guiado la construcción de nuestro instrumento de análisis en el que los indicadores que lo constituyen reúnen todos los ítems sugeridos por ellos, incluyendo aspectos relacionados con la problemática ambiental y con la calidad de vida, etc.

Igualmente nos han servido de un importante punto de apoyo a la hora de elaborar nuestro instrumento de análisis los documentos elaborados por Silva (2007) y Pereira (2012), ambos validados por especialistas en educación en ciencias, y centrados, respectivamente, en los principios de la educación CTSA y en la perspectiva de educación en ciencias promotora de cultura científica. Estos documentos han sido estudiados, discutidos y, posteriormente, reformulados y adaptados al fin deseado. 
El instrumento final se realizó basándonos en tres dimensiones de análisis: (1) Finalidades de la educación en ciencias relativas a la formación personal y social de los individuos así como al desarrollo de capacidades, actitudes y educación para la ciudadanía; (2) Conocimientos de ciencias relativos a la pertinencia, diversidad y naturaleza de temas y contenidos científicos considerados esenciales para los estudiantes; y (3) Procedimientos Metodológicos utilizados en ciencias para lograr los aprendizajes de los estudiantes, relacionados con las estrategias y actividades de la enseñanza. Cada una de estas tres dimensiones se compone de diferentes parámetros que, a su vez, integran un número variable de indicadores. Los parámetros representan las ideas-clave de cada dimensión a la que pertenecen los indicadores, expresando éstos la concretización de las interrelaciones CTSA. La selección de estas tres dimensiones - Finalidades, Conocimientos, Procedimientos Metodológicos - pretende dar respuesta a las tres cuestiones que constituyen las preocupaciones centrales de la educación en ciencias con enfoque CTSA: ¿Por qué enseñar ciencia? "Dimensión Finalidades"; ¿Que ciencia se debe enseñar? "Dimensión Conocimientos"; ¿Cómo se debe enseñar ciencia? "Dimensión Procedimientos Metodológicos". La Figura 1 representa la estructura que ha permitido construir el instrumento de análisis, así como las relaciones que se establecen entre las dimensiones, parámetros e indicadores.
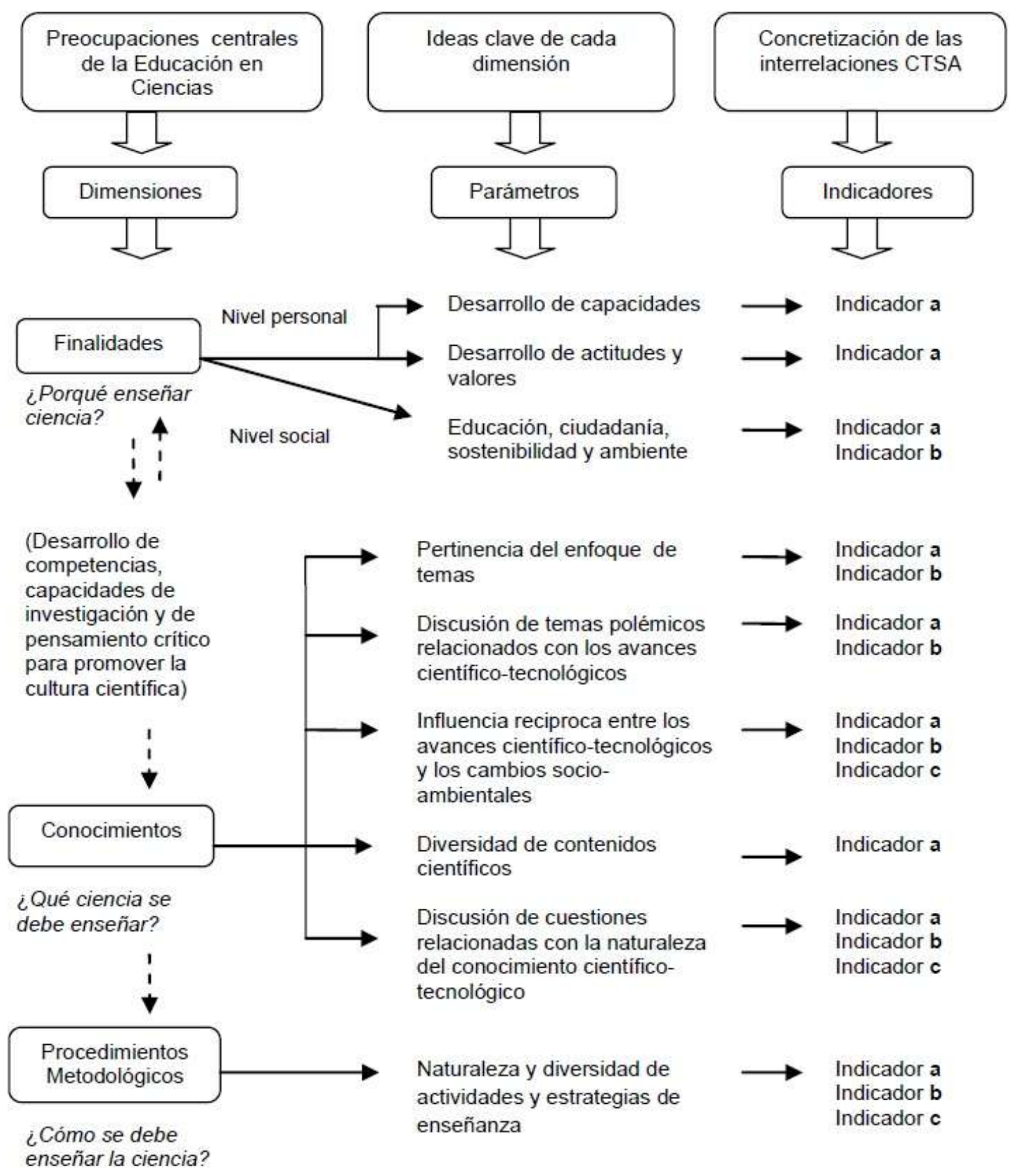

Pertinencia del enfoque de temas

Discusión de temas polémicos relacionados con los avances científico-tecnológicos

Influencia reciproca entre los avances científico-tecnológicos y los cambios socioambientales

\section{Diversidad de contenidos} científicos

Discusión de cuestiones relacionadas con la naturaleza del conocimiento cientificotecnológico

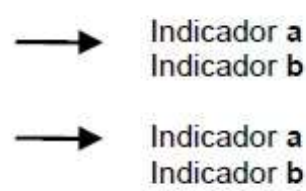

Naturaleza y diversidad de actividades y estrategias de enseñanza
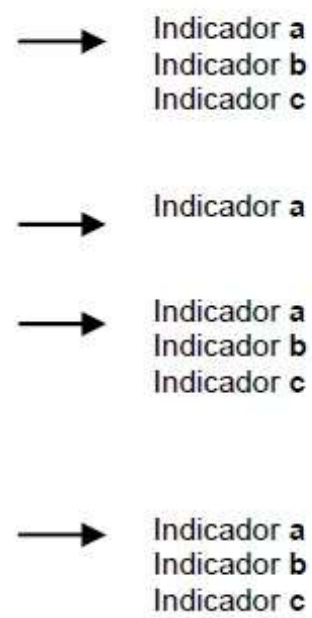

Fig. 1: Relaciones que se establecen entre las dimensiones, parámetros e indicadores del instrumento de análisis. 
Para asegurar la coherencia entre el instrumento y el fin establecido (análisis de documentos curriculares en el ámbito de la perspectiva CTSA), así como para garantizar su validez y fiabilidad, hemos recurrido a un grupo de jueces expertos en el área de la Didáctica de las Ciencias. Además, el instrumento fue presentado y debatido públicamente (Fernandes, Pires y Villamañán 2013) con objeto de obtener contribuciones y sugerencias de mejora, sometiéndolo a las recomendaciones y reformulaciones de otros investigadores en el campo de la Didáctica de las Ciencias. Los indicadores de cada dimensión/parámetro del instrumento de análisis se encuentran descritos en la Tabla 1.

Tabla 1: Instrumento de análisis de documentos curriculares desde la perspectiva CTSA

\begin{tabular}{|c|c|c|}
\hline Dimensión & Parámetros & Indicadores \\
\hline \multirow{3}{*}{ 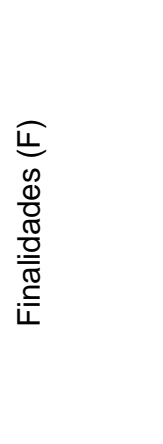 } & F.P1 - Desarrollo de Capacidades & $\begin{array}{l}\text { a. Propone el desarrollo de procedimientos científicos (observar, } \\
\text { inferir, clasificar, explicar, relacionar, argumentar...), la } \\
\text { resolución de problemas y la mejora del pensamiento crítico }\end{array}$ \\
\hline & $\begin{array}{l}\text { F.P2 - Desarrollo de actitudes y } \\
\text { valores }\end{array}$ & $\begin{array}{l}\text { a. Fomenta el desarrollo de principios y normas de conducta } \\
\text { responsables y conscientes, individuales y colectivas. }\end{array}$ \\
\hline & $\begin{array}{l}\text { F.P3 - Educación, ciudadanía, } \\
\text { sostenibilidad, y medio ambiente }\end{array}$ & $\begin{array}{l}\text { a. Promueve el desarrollo de decisiones conscientes, informadas } \\
\text { y argumentadas frente a las consecuencias de la acción humana } \\
\text { en el ambiente. } \\
\text { b. Fomenta el compromiso del estudiante en cuestiones } \\
\text { problemáticas actuales relacionadas con la ciudadanía, la } \\
\text { sostenibilidad y la protección del ambiente. }\end{array}$ \\
\hline \multirow{5}{*}{ 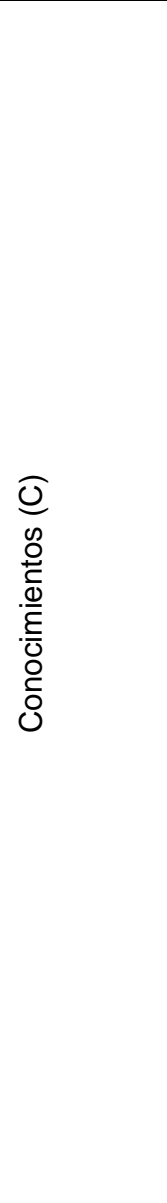 } & $\begin{array}{l}\text { C.P1 - Relacionados con el enfoque } \\
\text { de temas }\end{array}$ & $\begin{array}{l}\text { a. Sugiere el enfoque contextualizado de temas actuales, } \\
\text { relacionados con los conocimientos previos de los estudiantes y } \\
\text { con su vida cotidiana. } \\
\text { b. Propone la discusión de temas científicos en función de su } \\
\text { utilidad social. }\end{array}$ \\
\hline & $\begin{array}{l}\text { C.P2 - Discusión de temas polémicos } \\
\text { relacionados con los avances } \\
\text { científico-tecnológicos }\end{array}$ & $\begin{array}{l}\text { a. Analiza situaciones en que diferentes realidades sociales son } \\
\text { el origen de nuevos descubrimientos científicos e innovaciones } \\
\text { tecnológicas (cuestiones éticas, desigualdades } \\
\text { socioculturales...) } \\
\text { b. Trata las ventajas y los límites del conocimiento científico- } \\
\text { tecnológico, así como sus impactos en la sociedad y en el } \\
\text { ambiente. }\end{array}$ \\
\hline & $\begin{array}{l}\text { C.P3 - Influencia reciproca entre los } \\
\text { avances científico-tecnológicos y los } \\
\text { cambios socio-ambientales }\end{array}$ & $\begin{array}{l}\text { a. Pone de manifiesto las relaciones reciprocas entre la ciencia y } \\
\text { la tecnología. } \\
\text { b. Destaca los cambios en las condiciones de vida de las } \\
\text { personas (hábitos, estilo de vida, creación de nuevos recursos, } \\
\text { etc.) relacionadas con los avances tecnológicos a lo largo del } \\
\text { tiempo. } \\
\text { c. Enfatiza los impactos de la sociedad y del ambiente en los } \\
\text { avances científico-tecnológicos. }\end{array}$ \\
\hline & $\begin{array}{l}\text { C.P4 - Diversidad de contenidos } \\
\text { científicos }\end{array}$ & $\begin{array}{l}\text { a. Da prioridad al estudio de contenidos científico-tecnológicos } \\
\text { relacionados con otros campos del saber donde se exige la } \\
\text { comprensión de las inter-relaciones CTSA. }\end{array}$ \\
\hline & $\begin{array}{l}\text { C.P5 - Discusión de cuestiones } \\
\text { relacionadas con la naturaleza del } \\
\text { conocimiento científico-tecnológico }\end{array}$ & $\begin{array}{l}\text { a. Presenta datos relacionados con la naturaleza y la historia de } \\
\text { la ciencia y/o diferentes visiones del conocimiento científico a lo } \\
\text { largo de los tiempos; } \\
\text { b. Propone el conocimiento de una forma no dogmática; } \\
\text { c. Informa sobre el trabajo y función del científico, así como de } \\
\text { posibles presiones sociales, políticas, religiosas o económicas } \\
\text { que puede sufrir. }\end{array}$ \\
\hline 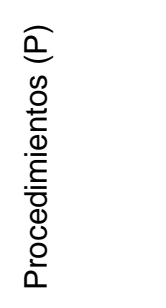 & $\begin{array}{l}\text { P.P1 - Naturaleza y diversidad de } \\
\text { actividades y estrategias de la } \\
\text { enseñanza }\end{array}$ & $\begin{array}{l}\text { a. Incita al estudiante para utilizar diferentes recursos dentro y } \\
\text { fuera del aula. } \\
\text { b. Propone la realización de actividades prácticas, } \\
\text { experimentales, de laboratorio, salidas de campo...para explorar } \\
\text { las relaciones CTSA } \\
\text { c. Involucra activamente al estudiante en actividades de } \\
\text { debates, resolución de problemas, discusiones, indagación } \\
\text { sobre cuestiones donde se manifieste la interacción CTSA. }\end{array}$ \\
\hline
\end{tabular}


Para facilitar la recogida y tratamiento de datos se han definido códigos de carácter acumulativo y secuencial, que permiten una identificación inmediata de cada evento que se pretende analizar. De esta manera, el primer código se refiere a la dimensión, siendo cada uno representado con la primera letra (ej.: $\mathrm{F}$ corresponde a la dimensión Finalidades). El segundo código representa el parámetro dentro de cada dimensión (ej.: F.P1 corresponde al primero parámetro - Desarrollo de Capacidades, integrado en la dimensión Finalidades). Por último, el tercer código se refiere al indicador(s) perteneciente a cada parámetro. Se representa por orden alfabético, en función del número de indicadores por parámetro (ej.: F.P1.a. corresponde al indicador a - Propone el desarrollo de procesos científicos, observar, inferir, clasificar, explicar, relacionar, argumentar..., la resolución de problemas y la mejora del pensamiento crítico, integrado en el parámetro Desarrollo de Capacidades, que integra la dimensión Finalidades). Esta codificación de datos permite identificar y recoger un conjunto de unidades de análisis/episodios, que hacen la etapa posterior de tratamiento, inferencial e interpretativo, más clara y objetiva y un análisis de contenido más riguroso y válido. Se presenta en la Tabla 1 la versión final del instrumento.

\section{Cómo Aplicar el instrumento}

Para comprobar la validez y fiabilidad del instrumento, se ha llevado a cabo un estudio piloto de una sección de las directrices curriculares de la Educación Primaria (10 - 12 años) de Portugal y de España. Hemos iniciado el estudio piloto identificando, en las directrices curriculares, unidades de análisis. Posteriormente, a través de un análisis cualitativo, se ha examinado la coherencia entre los indicadores del instrumento y el texto de los documentos analizados. En la etapa de tratamiento inferencial e interpretativo de los datos, se relacionaron las ideas de los indicadores del instrumento con las expresadas en las unidades de análisis identificadas. Veamos algunos ejemplos de la aplicación del instrumento al estudio piloto:

Unidad de análisis identificada en un documento curricular Portugués (ME - DGEBS, Vol. I, p.185), “...o ensino deverá ser problematizado, questionando as alternativas e o valor de soluções fornecidas pela Ciência...à medida que o ambiente sócio-cultural se altera e as técnicas de investigação melhoram, hipóteses e teorias bem estabelecidas podem ser desafiadas...substituídas". Este extracto es claro cuando afirma que la enseñanza debe ser problematizada y que las soluciones aportadas por la ciencia a lo largo del tiempo deben ser cuestionadas, evidenciando que el conocimiento científico tiene un carácter provisional. Además, el texto señala que esta provisionalidad del conocimiento científico se debe al cambio del entorno sociocultural y a la mejora de las técnicas de investigación, realzando el impacto que el cambio de la Sociedad y el Ambiente tienen en los avances del conocimiento científico-tecnológico. Estas ideas están de acuerdo con el indicador C.P5.b. (Propone el conocimiento de una forma no dogmática), del parámetro C.P5 (Discusión de cuestiones relacionadas con la naturaleza del conocimiento científicotecnológico) de la dimensión Conocimientos.

Unidad de análisis identificada en un documento curricular español (MEC, O. ECI, 2007, p. 31499), “Utilizar las tecnologías de la información y la comunicación para obtener información y como instrumento para aprender y compartir conocimientos, valorando su contribución a la mejora de las condiciones de vida de todas las personas". Es un objetivo propuesto en el documento en el cual la perspectiva CTSA está contemplada de forma explícita, al considerar que los estudiantes deben ser capaces de usar las tecnologías de comunicación y información, consideradas como una forma de obtener, aprender y compartir conocimiento, que contribuyen para cambiar y mejorar las condiciones de vida de las personas a lo largo del tiempo. Estas ideas están de acuerdo con el indicador C.P3.b. (Destaca los cambios en las condiciones de vida de las personas - hábitos, estilo de vida, creación de nuevos recursos, etc. - relacionadas con los avances tecnológicos a lo largo del tiempo), del parámetro C.P3 (Influencia reciproca entre los avances científico-tecnológicos y los cambios socio-ambientales) de la dimensión Conocimientos.

\section{CONCLUSIONES}

Para fomentar una adecuada educación científica, capaz de promover la cultura científica en los estudiantes es necesario que el currículo esté adaptado a las necesidades de la sociedad actual, por ello, consideramos que es importante poder poner de manifiesto si las recomendaciones de los documentos curriculares de la enseñanza de las ciencias, reguladoras de la acción de los profesores a nivel del aula, están de acuerdo con una educación CTSA (Ciencia, Tecnología, Sociedad, Ambiente).

Hemos construido un instrumento de análisis para poder examinar los documentos reguladores de la acción educativa, para cualquier nivel de la enseñanza, cuya concepción se ha fundamentado en datos y consideraciones de investigaciones recientes en Didáctica de las Ciencias. Para comprobar su validez y fiabilidad como instrumento de análisis se ha realizado un estudio piloto de una sección de las directrices curriculares de Educación Primaria (10 - 12 años) de Portugal y España. 
El instrumento desarrollado puede ayudar a poner de manifiesto si los documentos curriculares contemplan la perspectiva de enseñanza CTSA, en lo que concierne a: (a) las Finalidades de la enseñanza de las ciencias (relativas tanto a la formación personal y social de los individuos como al desarrollo de capacidades, actitudes y educación para la ciudadanía); (b) los Conocimientos de ciencias considerados esenciales para los estudiantes (entre los cuales es fundamental la presencia de las interacciones entre ciencia, tecnología, sociedad y ambiente; de temas polémicos y controvertidos sobre la ciencia, la tecnología y la sociología externa e interna de la ciencia, así como a la naturaleza del conocimiento científico-tecnológico); y (c) los Procedimientos Metodológicos utilizados en ciencias para fomentar el aprendizaje de los estudiantes (diferentes estrategias y actividades de la enseñanza como, por ejemplo, actividades de argumentación, debates, discusiones, indagación, etc., sobre cuestiones donde se manifieste la interacción CTSA).

\section{REFERENCIAS}

AAAS, Benchmarks for Science Literacy: A project 2061 report, New York: Oxford University Press, (1993).

AAAS, Science for all americans. A project 2061 report on literacy goals in Science, Mathematics, and Technology. New York: Oxford University Press, (1990).

Acevedo-Díaz, J.A., Vázquez-Alonso, A., Manassero-Mas, M.A. \& Acevedo-Romero, P. Consensos sobre la naturaleza de la ciencia: fundamentos de una investigación empírica. Revista Eureka sobre Enseñanza y Divulgación de las Ciencias, 4(1), 42-66, (2007).

Aikenhead, G. S., Educação científica para todos. Mangualde: Edições Pedago, (2009).

Aikenhead, G. S., Ryan, A. G. y Fleming, R. W., VOSTS - Views on Science-Technology-Society. Canada: University of Saskatchewan, (1989).

Alves, N., Recursos de Ensino/Aprendizagem para a implementação da perspetiva CTSA no 2. CEB. Tese de Mestrado, Escola Superior de Educação de Bragança, (2011).

Díaz, I. y García, M., Más Allá del Paradigma de la Alfabetización. La Adquisición de Cultura Científica como Reto Educativo, Form. Univ. 4(2), 3-14, (2011).

EURYDICE, Science Education in Europe: National Policies, Practices and Research, (2011), (documento en línea) http://eacea.ec.europa.eu/education/eurydice/documents/thematic_reports/133EN.pdf, Acceso: 12 de mayo (2013).

EURYDICE, Science Teaching in schools in Europe: Policies and Research, (2006), (documento en línea) http://eacea.ec.europa.eu/education/eurydice/index_en.php, Acceso: 12 de mayo (2013).

Fernandes, I.M. y Pires, D.M, As inter-relações CTSA nos manuais escolares de ciências do $2^{\circ}$ CEB. EDUSER: revista de educação, (documento en línea), 5(2), 35-47, (2013). https://www.eduser.ipb.pt/index.php/eduser/article/viewFile/120/84. Aceso: 9 de mayo (2014).

Fernandes, I.M.; Pires, D. M. y Villamañán, R.M., Educação em ciências com orientação CTSA Construção de um instrumento de análise das orientações Curriculares, Enseñanza de las Ciencias, CD, Número Extra IX Congreso Internacional sobre Investigación en Didáctica de las Ciencias, 459-462, Girona, España, (2013).

Filho, D.B, Maciel, M.D., Sepini, R.P. y Vázquez-Alonso, A., Alfabetização Científica sob o enfoque da Ciência, Tecnologia e Sociedade: Implicações para a formação inicial e continuada de professores. Revista Electrónica de Enseñanza de las Ciencias. 12(2), 313-333. (2013), (documento en línea) http://reec.uvigo.es/volumenes/volumen12/reec_12_2_5_ex649.pdf, Acceso: 8 de octubre (2013).

García-Carmoma, A. y Criado, A. M., Naturaleza de la Ciencia en Educación Primaria: Análisis de su presencia en el currículo oficial español, VII Seminario Ibérico/III Seminario Iberoamericano CTS en la enseñanza de las Ciencias "Ciencia, Tecnología y Sociedad en el futuro de la enseñanza de las ciencias", Madrid, 28 al 30 de septiembre (2012).

Gil, D. y Vilches, A. Contribución de la ciencia a la cultura ciudadana. Cultura y Educación, 16 (3), 259-272, (2004). 
Gonzalez, L.M. y Rasilla M., Una estrategia para el Aprendizaje de la Cultura Científica. Form. Univ. 4(2), 15-26, (2011).

León, M.P.; Colón, A.O. y Alvarado, F.C., ¿Cómo promover la educación científica en el alumnado de primaria? Una experiencia desde el contexto Ecuatoriano. Revista Eureka sobre Enseñanza y Divulgación de las Ciencias 10(2), 199-209, (2013).

Manassero, M. A., Vázquez, A. y Acevedo, J. A., Cuestionario de Opiniones sobre Ciencia, Tecnología y Sociedad (COCTS). Princeton, NJ: Educational Testing Service, (2003). (documento en línea) http://www.ets.org/testcoll/. Acceso: 10 de marzo (2013).

Manassero, M.A.; Vázquez, A. y Acevedo, J. A., Avaluació dels temes de ciència, tecnologia $i$ societat. Palma de Mallorca: Conselleria d’Educació i Cultura del Govern de les Illes Ballears, (2001).

Manassero-Mas, M.A.; Roig, B.; Bonnin, S.O. y Moralejo, R.O., Innovar la educación en ciencias a través de enseñar y aprender acerca de la naturaleza de ciencia y tecnología. Enseñanza de las Ciencias, CD, Número Extra IX Congreso Internacional sobre Investigación en Didáctica de las Ciencias Girona, España, 2103-2108, (2013).

Martins, I., Abelha, M., Costa, N. y Roldão, M., Impacto do currículo português das ciências físicas e naturais nas práticas docentes. Ciência \& Educação (Bauru), 17 (4), 771-788, (2011), (documento en línea) http://www.redalyc.org/src/inicio/ArtPdfRed.jsp?iCve=251021295001, Acceso: 12 de mayo (2013).

MEC: ORDEN ECI/2211/2007, de 12 de julio, por la que se establece el currículo y se regula la ordenación de la Educación Primaria. Boletín Oficial del Estado, no 173, de 20 de julio de 2007, Ministerio de Educación y Ciencia, Gobierno de España, 31487-31566, (2007).

Millar, R. y Osborne, J., Beyond 2000: Science education for the future. London: King's College London, School of Education, (1998).

Ministério da Educação - DGEBS, Ensino Básico 2. ciclo: Organização Curricular e programas, (Volume I). Lisboa: Imprensa Nacional, (1991).

National Research Council (NRC), National Science Education Standards. Washington, DC: National Academic Press, (1996).

OEI (Organização dos Estados iberoamericanos), Memoria de la programación 1999-2000, 121-134, Madrid, (2001), (documento en línea) http://www.oei.es/, Acceso: 5 de enero (2012).

Osborne, J. y Dillon, J., Science Education in Europe: Critical Reflections - A Report to the Nuffield Foundation, 2008, (documento en línea) http://www.polleneuropa.net/pollen_dev/Images_Editor/Nuffield\%20report.pdf. Acceso: 5 de enero (2012).

Pereira, J.S., Educação em ciências em contexto pré-escolar. Tese de doutoramento. Departamento de Educação, Universidade de Aveiro, (2012).

Prieto, T., España, E. y Martín, C., Algunas cuestiones relevantes en la enseñanza de las ciencias desde una perspectiva Ciencia-Tecnología-Sociedad. Revista Eureka sobre Enseñanza y Divulgación de las Ciencias, 9(1), 71-77, (2012).

Rocard, M. et al (High Level Group on Science Education), Science Education Now: a Renewed Pedagogy for the Future of Europe, Bruxelas, (2007).

Rodrigues, M. J. y Vieira, R.M., Programa de formação de educadoras de infância: Seu contributo para a (re)construção de conceções Ciência-Tecnologia-Sociedade. Revista Electrónica de Enseñanza de las Ciencias, 3 (11), 501-520, (2012).

Silva, A.M., Educação em Ciências no 1ํ CEB: Desenvolvimento de Competências em Contextos CTSA. Dissertação de mestrado, Departamento Didática e Tecnologia Educativa, Universidade de Aveiro, Aveiro, (2007). 
Vieira, N., Literacia Científica e Educação de Ciências. Dois objetivos para a mesma aula. Revista Lusófona da Educação, 10, 97-108, (2007).

Vieira, R. M., Tenreiro-Vieira, C. y Martins, I. P., A educação em ciências com orientação CTS - atividades para o ensino básico. Porto: Areal Editores, (2011).

Vilches, A. y Gil-Pérez, Educación Ambiental y Educación para el Desarrollo Sostenible: Convergencias y (supuestas) divergencias. In Universidade de Brasília (Ed.), Educação para uma nova ordem socioambiental no contexto da crise global. II Seminário Ibero-americano Ciência-Tecnologia-Sociedade no Ensino das Ciências (VI Seminário Ibérico CTS no Ensino das Ciências). Brasília: Universidade de Brasília, (2010). 